OPEN ACCESS

Edited by: Fumiyo Ikeda

Kyushu University, Japan

Reviewed by:

Yenniffer Ávalos,

University of Santiago, Chile

Magdalena Paolino,

Karolinska Institutet (KI), Sweden

*Correspondence:

Jacki Kornbluth

jacki.kornbluth@health.slu.edu

Specialty section:

This article was submitted to

Integrative Physiology,

a section of the journal

Frontiers in Physiology

Received: 16 June 2020 Accepted: 05 October 2020 Published: 29 October 2020

Citation:

Lawrence DW, Willard PA, Cochran AM, Matchett EC and Kornbluth J (2020) Natural Killer Lytic-Associated Molecule (NKLAM): An E3 Ubiquitin Ligase With an Integral Role in Innate Immunity.

Front. Physiol. 11:573372 doi: 10.3389/fphys.2020.573372

\section{Natural Killer Lytic-Associated Molecule (NKLAM): An E3 Ubiquitin Ligase With an Integral Role in Innate Immunity}

\author{
Donald W. Lawrence ${ }^{1}$, Paul A. Willard ${ }^{1}$, Allyson M. Cochran ${ }^{1}$, Emily C. Matchett ${ }^{1}$ and \\ Jacki Kornbluth ${ }^{1,2 *}$
}

${ }^{1}$ Department of Pathology, Saint Louis University School of Medicine, St. Louis, MO, United States, ${ }^{2}$ St. Louis VA Health Care System, St. Louis, MO, United States

Natural Killer Lytic-Associated Molecule (NKLAM), also designated RNF19B, is a unique member of a small family of E3 ubiquitin ligases. This 14-member group of ligases has a characteristic cysteine-rich RING-IBR-RING (RBR) domain that mediates the ubiquitination of multiple substrates. The consequence of substrate ubiquitination varies, depending on the type of ubiquitin linkages formed. The most widely studied effect of ubiquitination of proteins is proteasome-mediated substrate degradation; however, ubiquitination can also alter protein localization and function. Since its discovery in 1999, much has been deciphered about the role of NKLAM in innate immune responses. We have discerned that NKLAM has an integral function in both natural killer (NK) cells and macrophages in vitro and in vivo. NKLAM expression is required for each of these cell types to mediate maximal killing activity and cytokine production. However, much remains to be determined. In this review, we summarize what has been learned about NKLAM expression, structure and function, and discuss new directions for investigation. We hope that this will stimulate interest in further exploration of NKLAM.

Keywords: NKLAM, ubiquitin ligase, innate immunity, natural killer, macrophage, phagocytosis, cytotoxicity, RNF19B

\section{INTRODUCTION TO NKLAM}

\section{Discovery of NKLAM}

Studies were initiated to identify new genes and gene products associated with cytokine-enhanced natural killer (NK) anti-tumor cytotoxic activity. For these experiments, we used the human NK clone NK3.3, which had been generated previously (Kornbluth et al., 1982). This cell line was cloned from the peripheral blood of a healthy individual, and has all the characteristics of an NK cell. Most importantly, the cytotoxic activity of NK3.3 can be upregulated by cytokine stimulation (Kornbluth and Hoover, 1988). A cDNA library from interferon beta (IFN $\beta$ ) stimulated NK3.3 cells was made and differential screening was performed to compare expression in unstimulated cells. From this analysis, 56 IFN $\beta$-upregulated genes were identified; 46 were novel at the time. We named one of those novel cDNA clones Natural Killer Lytic-Associated Molecule (NKLAM) (Kozlowski et al., 1999).

Kinetic analysis determined that NKLAM mRNA levels peak 4-6 $\mathrm{h}$ after IFN $\beta$ stimulation of NK cells; additionally, NKLAM levels are strongly induced by interleukin (IL)-2, peaking 
6-12 h after IL-2 stimulation. These expression levels strongly correlate with both IFN $\beta$ and IL-2-enhanced NK3.3 anti-tumor cytolytic activity. NKLAM mRNA is short lived, with a half-life of 2.5 h (Kozlowski et al., 1999).

Peripheral blood subsets from healthy donors were isolated and examined for NKLAM mRNA expression. Levels were found to be relatively high in monocytes and upregulated by IFN $\beta$. NKLAM mRNA levels were also high in NK cells and further upregulated by IL-2 and IFN $\beta$. NKLAM mRNA was not found in resting $\mathrm{T}$ cells and not induced by IL2 or the mitogen phytohemagglutinin (PHA). NKLAM was, however, expressed in a $\mathrm{CD}^{+}$cytotoxic $\mathrm{T}$ lymphocyte (CTL) clone and further upregulated by exposure to its target antigen (Kozlowski et al., 1999).

\section{NKLAM Gene Structure}

We cloned both full-length human and mouse NKLAM (Portis et al., 2000). NKLAM is highly conserved throughout evolution. There is $89 \%$ nucleotide and $94 \%$ amino acid homology between human and mouse NKLAM. The human NKLAM gene is composed of 9 exons and is found on chromosome 1 (Figure 1). Mouse NKLAM has an almost identical genetic structure of 9 exons and maps to chromosome 4. A major difference is that in humans, there are two forms of NKLAM protein: one is 732 amino acids and the other is 587 amino acids. These arise by alternative splicing in exon 9, resulting in two mRNA transcripts that differ at the $3^{\prime}$ end. Mice (and rats) only produce one mRNA transcript, encoding the longer form of NKLAM protein (Portis et al., 2000). One unresolved question is the functional difference between the two forms of human NKLAM. Both transcripts and proteins are similarly up-regulated and expressed upon activation of human NK cells and macrophages.

\section{NKLAM Protein Structure and Homology to RING-In Between RING-RING (RBR) Ubiquitin Ligases}

When we first described NKLAM, computer analysis predicted it to be a transmembrane protein. It also identified three cysteinerich clusters, with homology to proteins found in Caenorhabditis elegans, mosquitos and in the mouse ovary. This cysteine-rich domain is $99 \%$ identical between mouse and human NKLAM. Subsequent cloning and characterization of additional proteins with this signature domain placed NKLAM within the E3 RBR ubiquitin ligase family of proteins. Ubiquitination is one of the most important post-translational modifications, regulating both the stability, function and localization of proteins. Ubiquitin ligases modify proteins by adding either a single ubiquitin or polyubiquitin chains (Swatek and Komander, 2016). Over 800 ubiquitin ligases are known; only 14 have this RBR structure (Smit and Sixma, 2014; Spratt et al., 2014; Lechtenberg et al., 2016; Dove and Klevit, 2017). In addition, only 4 of these are transmembrane proteins like NKLAM. All the RBR E3 ubiquitin ligases are highly conserved and important in cellular physiology. The most studied RBR ligase is Parkin; mutations in Parkin are associated with autosomal recessive juvenile Parkinson's disease (Kitada et al., 1998). By their ability to control expression of key regulatory proteins involved in cell growth and death signaling, ubiquitin ligases also have a role in development of autoimmunity and cancer (Sun, 2006).

Ubiquitination is a three-step process that involves an E1 activating enzyme that uses ATP and a catalytic cysteine to activate ubiquitin, an E2 conjugating enzyme that accepts the activated ubiquitin from the E1, and an E3 ligase that coordinates the ligation of the E2-bound active ubiquitin onto the target protein (Hochstrasser, 1996). There are three families of E3 ubiquitin ligases: HECT-type, RING-type, and RBR-type ligases. HECT ligases contain the HECT (homologous to the E6-AP carboxyl terminus) domain which provides a catalytic cysteine to accept active ubiquitin from the E2 and form an intermediate E3-ubiquitin prior to ligation onto the substrate (Weber et al., 2019). This frees the E2 and enables the HECT E3 ligase to dictate whether the ubiquitin is added singly to a target substrate or ligated to create a ubiquitin chain. RING ligases are identified by the presence of a RING (Really Interesting New Gene) domain of the canonical C3HC4 structure. RING ligases do not have a catalytic cysteine, but function as a scaffold, facilitating the direct transfer of the active ubiquitin from the E2 to the substrate (Metzger et al., 2014). It is, therefore, the E2 that determines the ubiquitin linkage that is created. RBR ligases are composed of a C3HC4 RING domain (RING1), identical to RING-type ligases, followed by an In-Between RING (IBR), and another RING (RING2) domain. RING2 contains a conserved catalytic cysteine for accepting active ubiquitin to form an intermediate E3-ubiquitin, similar to HECT-type ligases (Spratt et al., 2014; Walden and Rittinger, 2018). RBR ligases control ubiquitin linkage type in a manner similar to HECT ligases.

The conserved catalytic cysteine in the RING2 domain of RBR E3 ubiquitin ligases, including Parkin and Dorfin (RNF19A), is considered a defining feature of this ligase family. NKLAM also has a comparable cysteine (C302) that we predict is critical for its ligase activity (Lechtenberg et al., 2016; Dove and Klevit, 2017).

RING-IBR-RING ubiquitin ligases also have preference for the E2s that they interact with. We identified the ubiquitin conjugating enzymes $\mathrm{UbcH7}$ and $\mathrm{UbcH} 8$ interacting with NKLAM. There is a level of E2 specificity; UbcH10 is unable to bind NKLAM (Fortier and Kornbluth, 2006). Upon stimulation of NK cells with IFN $\beta$, both NKLAM and UbcH8 are upregulated and can be co-immunoprecipitated. These ubiquitin conjugates bind to the RING domain of NKLAM. Most E2-conjugating enzymes transfer ubiquitins onto both lysine and cysteine residues and therefore function with multiple types of ubiquitin ligases. However, $\mathrm{UbcH7}$ is unique in being strictly cysteinereactive and works predominantly with RBR E3 ligases like NKLAM (Martino et al., 2018).

The first 100 amino acids of NKLAM consist primarily of alanine, arginine, glutamate, glycine, and proline. Bioinformatic analysis using the DEPICTER server identifies this amino acid stretch as an intrinsically disordered region (IDR) of the protein (Barik et al., 2020; Figure 2). This combination and arrangement of amino acids is predicted to give NKLAM the capability to bind RNA, DNA, and protein. Following the N-terminal IDR is the RBR ligase domain of NKLAM. There are two transmembrane domains that anchor the protein, and a second larger IDR at the 


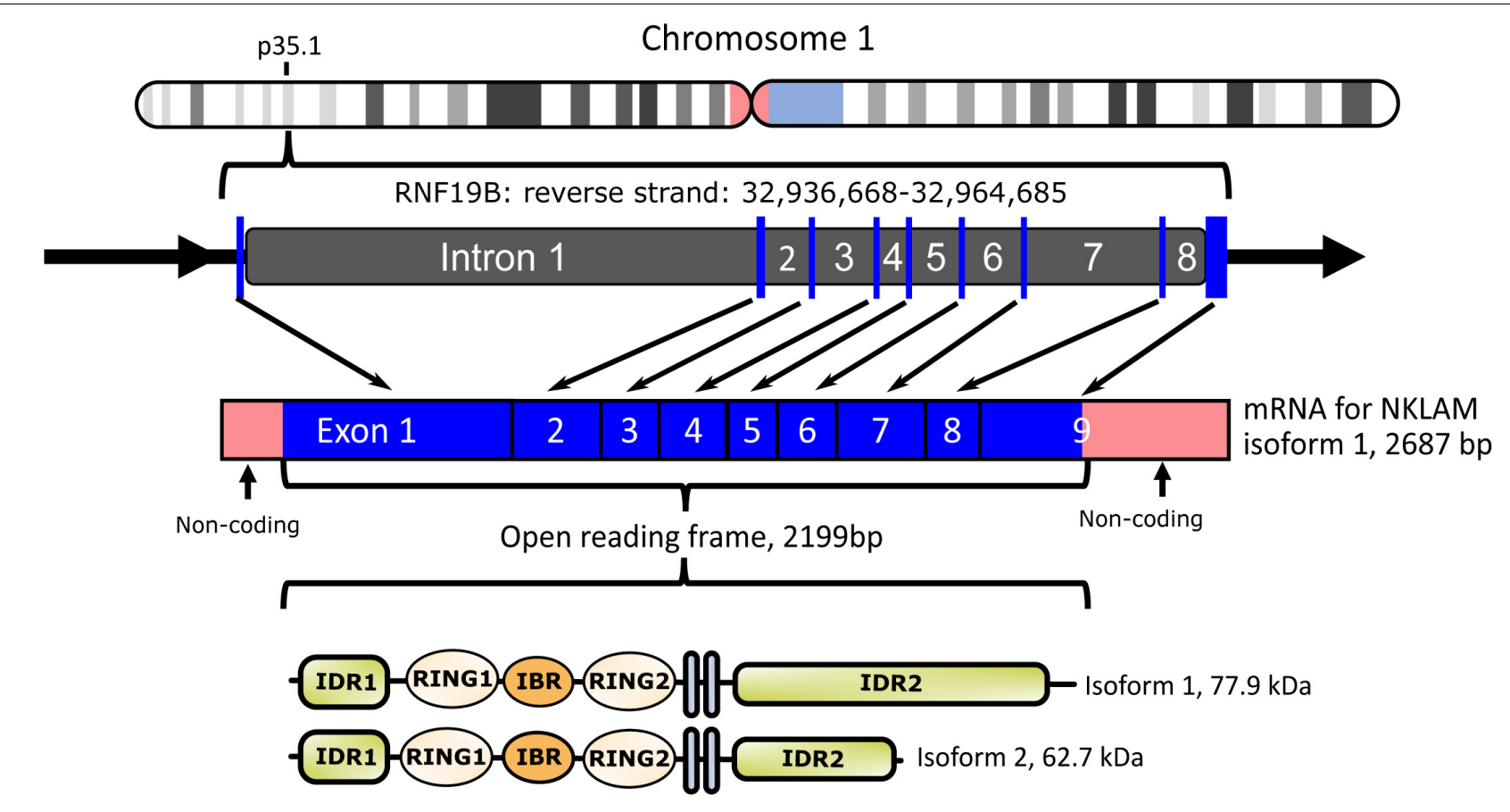

FIGURE 1 | Diagram of human NKLAM (RNF19B) gene organization. NKLAM is encoded by 9 exons (depicted as blue bars) spanning $28 \mathrm{~kb}$ in the reverse strand of chromosome 1. Exons 1-3 encode intrinsically disordered region 1 (IDR1) and the three RINGS of the ligase domain. Exons 4 and 5 encode the two transmembrane domains (gray bars), while IDR 2 is encoded across the remaining exons 6-9. Mouse NKLAM, located on chromosome 4, has the same genomic structure. Human NKLAM mRNA isoform 1 encodes a protein with a predicted molecular weight of $77.9 \mathrm{kDa}$. Alternative splicing of exon 9 results in a shorter mRNA isoform 2 encoding a protein with a predicted molecular weight of $62.7 \mathrm{kDa}$. Mice only express the long form of NKLAM protein.

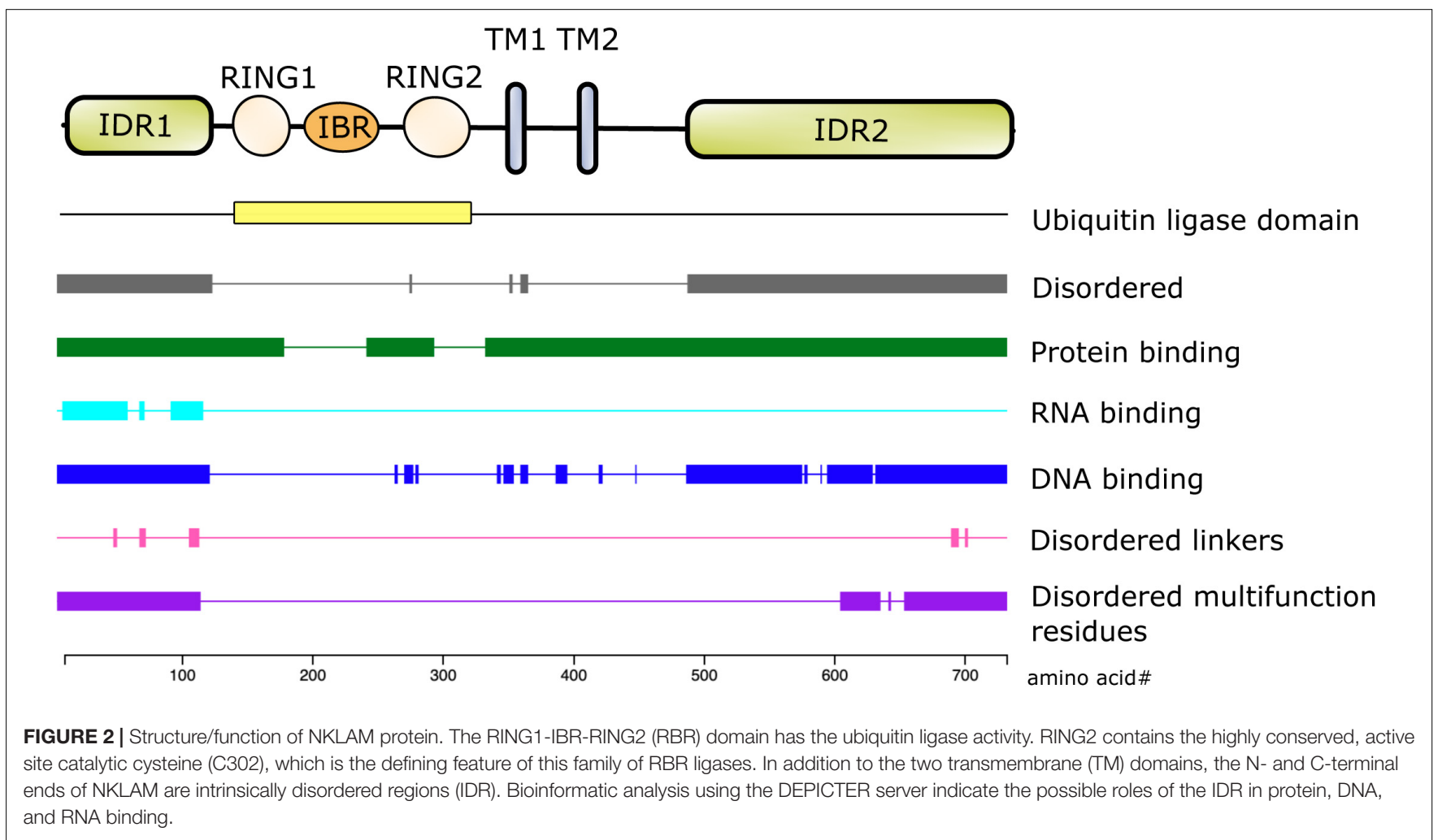


C-terminal end of NKLAM that is also predicted to interact with DNA and proteins.

Intrinsically disordered regions are typically rich in charged or polar residues, enabling interactions with proteins or nucleic acids (Wright and Dyson, 2015; Dyson, 2016). These regions are often flexible enough to either conform to the surface of an interacting protein, thereby stabilizing the interaction, or to adopt a different conformation to allow interactions with additional ligands (Dunker et al., 2005). Post-translational modifications of these regions can induce changes in conformation, thereby changing the accessibility of binding sites or altering the activity of the protein (Darling and Uversky, 2018; Owen and Shewmaker, 2019).

\section{Regulation of NKLAM Expression}

Natural killer lytic-associated molecule is expressed by a variety of hematopoietic cells, including NK cells, $\mathrm{CD}^{+}$cytotoxic $\mathrm{T}$ cells and murine bone marrow-derived macrophages (BMDM). Our earliest studies of NKLAM demonstrated its expression in freshly isolated human peripheral blood monocytes and upregulation by IFN $\beta$. Similar results were obtained using macrophages from the spleen and peritoneal cavity of mice (Portis et al., 2000). There is limited experimental data on NKLAM expression in non-immune cells; however, NKLAM was found to be associated with ppp1cc in testes (Fardilha et al., 2011). Our laboratory found that NKLAM is expressed in mouse tracheal epithelial cells treated with IFN $\gamma$ (Lawrence et al., 2019). The experimental data on NKLAM expression is largely derived from NK cell and macrophage studies.

Natural killer lytic-associated molecule expression is induced by both IFN $\gamma$ and IFN $\beta$; thus, NKLAM is an interferonstimulated gene (ISG). Resting NK cells have low levels of NKLAM; treatment with IFN $\beta$ rapidly induces transcription and translation of NKLAM, where it localizes to lytic granule membranes (Kozlowski et al., 1999). In addition to IFN $\beta$, IL-2, IL-12, IL-15, and IL-21 also promote transcription of NKLAM in NK cells. Similarly, under baseline conditions, macrophages express minimal amounts of NKLAM. Treatment with IFN $\gamma$ induces NKLAM expression in a time dependent manner with maximal levels reached at $12 \mathrm{~h}$ (Portis et al, 2000; Lawrence and Kornbluth, 2012). Using transcription factor binding site prediction software $\mathrm{PROMO}^{1}$, the promoter region of NKLAM contains a binding site for STAT1. The signal transduction pathway for IFN $\gamma$, a type II interferon, results in STAT1 binding a gamma-activated sequence (GAS) within an ISG promoter region. Additionally, there are STAT4 and STAT5 binding sites in the NKLAM promoter. STAT4 and STAT5 are involved in signal transduction from various cytokine receptors for IL-2, IL-12, and IL-15. The NKLAM promoter also contains binding sites for members of the interferon-regulatory factor (IRF) family of transcription factors. IRF proteins are regulators of the type I interferon system. The expression of NKLAM in mouse immune cells is the same as seen in human cells and its regulation is also mediated by the same activation signals.

\footnotetext{
${ }^{1}$ http://alggen.lsi.upc.es/cgi-bin/promo_v3/promo/promoinit.cgi?dirDB=TF_8.3/
}

Natural killer lytic-associated molecule expression can also be induced by Toll-like receptor (TLR) agonists. Mouse macrophage cell lines RAW 264.7 and J774A.1 were stimulated with TLR4 agonist lipopolysaccharide (LPS), the combination of LPS plus IFN $\gamma$, Escherichia coli, or Staphylococcus aureus and NKLAM protein expression was assessed over time. In both RAW 264.7 and J774 cells, peak NKLAM expression is seen $16 \mathrm{~h}$ after stimulation with all treatments (Lawrence and Kornbluth, 2012). Stimulation with LPS plus IFN $\gamma$ leads to the largest increase in NKLAM levels (Portis et al., 2000). Poly (I:C), a mimetic of double stranded RNA and a TLR3 agonist, was also found to induce NKLAM expression in BMDM (Lawrence et al., 2019). TLR stimulation culminates in the activation of transcription factors NFKB, IRFs, and MAP kinases to regulate gene expression (Kawasaki and Kawai, 2014). In addition to binding sites for IRF1 and IRF-2, the NKLAM promoter contains binding sites for $\mathrm{NF \kappa B}$ proteins $\mathrm{p} 50$ and $\mathrm{p} 65 /$ RelA.

\section{NKLAM FUNCTION}

\section{NKLAM Function in Cytotoxic Cells}

Natural killer lytic-associated molecule mRNA expression strongly correlates with cytotoxic activity. To determine whether NKLAM is necessary for NK killing, NK cells were treated with NKLAM antisense (AS) oligonucleotides (ODN) or control ODN. There is a significant and specific downregulation of NKLAM expression and cytotoxic function of NK cells after treatment with NKLAM AS ODN. Granule exocytosis-mediated killing is diminished after NKLAM AS ODN treatment; however, Fas-mediated killing appears to be NKLAM-independent. In $4 \mathrm{~h}$ killing assays, the cytotoxic activity of CTL against its specific target is reduced by $60 \%$ after treatment with NKLAM AS ODN (Kozlowski et al., 1999). This suggests that NKLAM function is associated with killing mediated by lymphocyte granule exocytosis.

We generated a panel of monoclonal antibodies to NKLAM. Resting peripheral blood NK cells express little to no NKLAM; upon cytokine activation, NKLAM protein levels increase. Subcellular fractionation experiments localized NKLAM to the membranes of the cytolytic granules in NK cells. Unlike other granule proteins, NKLAM is not pre-formed; it is rapidly transcribed, translated and embedded in granule membranes upon NK activation (Kozlowski et al., 1999).

To further investigate the function of NKLAM in NK cytotoxic activity, we generated NKLAM-deficient $\left(\mathrm{NKLAM}^{-/-}\right)$mice (Hoover et al., 2009). This was accomplished by genomic deletion of exons 2-5, which removes the 2nd and 3rd RING domains and both transmembrane domains. These mice are completely NKLAM deficient. By both RT-PCR and protein analysis, there is no detectable full length or fragments of NKLAM in these mice. Mice were backcrossed to C57BL/6 mice for 11 generations. $\mathrm{NKLAM}^{-/-}$mice appear normal and are fertile. The lymphoid and myeloid subpopulations in the spleen are comparable to wild type (WT) mice in numbers and distribution. They also have normal numbers of NK cells in the spleen; these NK cells have similar amounts of cytotoxic proteins perforin and granzyme B in 
their granules as WT NK cells. Granule release is comparable in both $\mathrm{NKLAM}^{-/-}$and WT NK cells. However, NKLAM ${ }^{-/-}$NK cells have $60 \%$ less tumor killing activity in vitro and secrete less IFN $\gamma$ after target or cytokine stimulation (Hoover et al., 2009).

\section{NKLAM-Mediated Ubiquitination of Uridine-Cytidine Kinase Like-1 (UCKL-1)}

We employed the yeast-two-hybrid system to identify potential substrate proteins that bind to and are ubiquitinated by NKLAM. The RING domain of NKLAM was used as bait to trap binding proteins from a human spleen cell cDNA library in yeast. Using high stringency binding conditions, we identified uridine-cytidine kinase-like 1 (UCKL-1, previously called URKL1) interacting with NKLAM. Co-transfection studies in HEK293 cells confirmed the interaction between NKLAM and UCKL-1, leading to UCKL-1 ubiquitination and degradation (Fortier and Kornbluth, 2006). NKLAM constructs containing one or more of the RING domains were designed and analyzed for their ability to bind UCKL-1 and ubiquitin conjugates. We demonstrated that the entire RBR domain, without the $\mathrm{N}$-terminal and C-terminal regions of NKLAM, is capable of binding UCKL-1, and ubiquitin conjugates $\mathrm{UbcH7}$ and $\mathrm{UbcH}$. Although UCKL-1 co-immunoprecipitates with multiple combinations of two of the three RING domains, it is maximally ubiquitinated and degraded by full length NKLAM or the entire RBR domain (Fortier and Kornbluth, 2006). Since the highly conserved catalytic cysteine (C302) in the RING2 domain is considered required for its ubiquitin ligase activity, we generated NKLAM constructs with a cysteine to alanine (C-A) mutation of C302 (C302A). A comparison of WT and mutant NKLAM will determine the role of ubiquitination in NKLAM functions.

Uridine kinases are involved in the pyrimidine salvage pathway. UCKL-1 is often upregulated in cancers and is proposed as a biomarker for several cancer types (Geiger et al., 2012; Cheng et al., 2014). Since uridine kinase expression is associated with cancer growth, we hypothesized that upon NK-tumor cell interaction, during the process of granule exocytosis, NKLAM may be able to interact with tumor-associated proteins, like UCKL-1. This would result in loss of UCKL-1 expression in the tumor cell, thereby impacting its survival.

To model the potential effect of NKLAM on reduction of UCKL-1 expression in tumor cells, we performed siRNA experiments. Downregulation of UCKL-1 in tumor cells by siRNA slows their proliferation, induces apoptosis and enhances their susceptibility to NK-mediated lysis (Ambrose and Kornbluth, 2009). Conversely, over-expression of UCKL1 protects tumor cells from NK killing and enhances tumor survival in vitro and in vivo (Gullickson et al., 2016). These data suggest a model where, upon NK-tumor interaction and release of lytic granules, NKLAM enters the tumor cell, ubiquitinates and degrades UCKL-1, thereby promoting cell death.

The cytotoxic granules in NK cells are heterogeneous, varying in size and in the amount of electron-dense material in their core. They are called hybrid organelles, with the properties of both lysosomes and secretory granules. This heterogeneity may reflect a continuum of maturation (Burkhardt et al., 1990;
De Saint Basile et al., 2010). Some of the granules contain multivesicular bodies, where smaller membrane-bound intragranular vesicles, exosomes, are generated. It has been demonstrated that exosomes derived from NK cells contain the cytolytic molecules perforin, granzymes $\mathrm{A}$ and $\mathrm{B}$ and granulysin, as well as the membrane proteins FasL and CD63, and have antitumor cytotoxic activity (Lugini et al., 2012; Jong et al., 2017). During NK-tumor cell interaction and degranulation, exosomes and other extracellular vesicles (EVs) are released, and may participate in tumor killing (Wu et al., 2019). This would provide a mechanism for delivery of the granule membrane protein NKLAM from the NK cell to the target cell, where it would ubiquitinate and degrade UCKL-1, ultimately resulting in cell death. A model of tumor killing is depicted in Figure 3.

\section{Role of NKLAM in Anti-Tumor Activity in vivo}

The B16 melanoma model of experimental lung metastasis was used to evaluate $\mathrm{NK}$ function in $\mathrm{NKLAM}^{-/-}$mice in vivo. This model is widely used in studies of NK function in vivo because B16 melanoma cells express low amounts of MHC class I molecules, are poorly immunogenic so they do not generate a significant adaptive immune response, and are readily killed by NK cells (Hanna and Burton, 1981; Gorelik et al., 1982; Warner and Dennert, 1982). NKLAM ${ }^{-/-}$and WT mice were injected intravenously with B16 melanoma cells; tumor colonies in the lungs were counted 15 days later. $\mathrm{NKLAM}^{-/-}$mice have substantially higher numbers and larger lung melanoma nodules than WT mice. These results indicate the importance of NKLAM in controlling tumor metastasis, likely by enhancing NK anti-tumor function (Hoover et al., 2009).

The role of NKLAM in NK-mediated tumor immunity in vivo was further investigated by employing additional tumor models to compare tumor development, progression and metastasis in $\mathrm{NKLAM}^{-/-}$and WT mice. We injected mice with RMA-S, a mouse T cell lymphoma, and well-characterized NK-susceptible hematopoietic tumor. These cells are MHC class I negative and are killed exclusively by NK cells (Kim et al., 2000; Cerwenka et al., 2001; Diefenbach et al., 2001). Using a sensitive realtime quantitative PCR assay for tumor burden, we found greater dissemination of RMA-S tumor cells to the lungs, lymph nodes, bone marrow and blood of NKLAM ${ }^{-/-}$mice compared to WT mice (Hoover et al., 2012). These results indicate that $\mathrm{NKLAM}^{-/-}$mice are less capable of controlling lymphoma dissemination than WT mice.

The potential role of NKLAM in controlling breast cancer growth and metastasis was evaluated by injecting syngeneic EO771 breast cancer cells into the mammary fat pads of $\mathrm{NKLAM}^{-/-}$and WT mice. EO771 is poorly immunogenic, incapable of generating a primary cytotoxic $\mathrm{T}$ cell response in vivo. It is also estrogen receptor positive, highly aggressive, and prone to metastasis (Ewens et al., 2005; Zhou et al., 2005; $\mathrm{Gu}$ et al., 2009). Primary tumor growth is similar between $\mathrm{NKLAM}^{-/-}$and WT mice. However, there are much higher levels of disseminated tumor cells in the bloodstream and more metastatic EO771 breast cancer cells in the lungs of NKLAM ${ }^{-/-}$ 


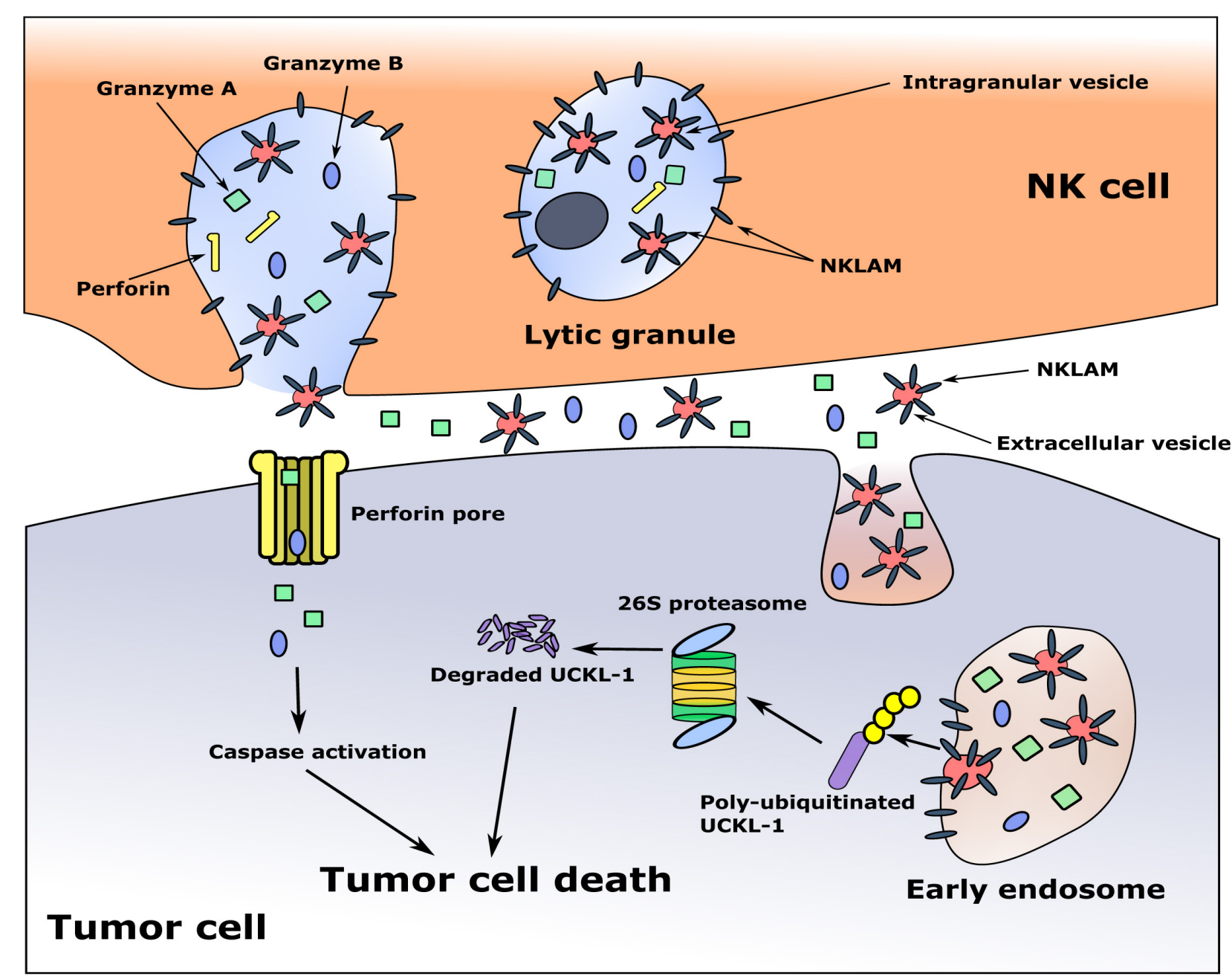

FIGURE 3 | Proposed model of NKLAM function in NK cells. The cytolytic granules of resting NK cells contain little to no NKLAM. Upon cytokine exposure or target stimulation, activated NK cells rapidly transcribe and translate NKLAM, which is embedded in cytolytic granule membranes. Smaller, intragranular vesicles also have NKLAM in their membranes. NK-tumor interaction releases granule contents (perforin, granzymes) into the tumor cell at the immunological synapse. Intragranular vesicles are also released; these now called extracellular vesicles (EVs) contain NKLAM, as well as perforin and granzymes, and enter the tumor cell by endocytosis. Granzymes induce caspase activation; NKLAM ubiquitinates and degrades the tumor survival protein UCKL-1. This combination of perforin, granzymes, and NKLAM ensures death of the tumor cell. Red circles: EVs; gray bars: NKLAM; green squares, blue ovals: cytolytic proteins granzymes A and B; yellow bars: perforin; purple bar: UCKL-1; yellow circles: ubiquitin.

than WT mice (Hoover et al., 2012). These results suggest that NKLAM-expressing NK cells play a key role in controlling tumor dissemination and metastasis in vivo. Reversal of these effects by reconstitution of $\mathrm{NKLAM}^{-/-}$mice with WT bone marrow would confirm the role of $\mathrm{NKLAM}^{+}$hematopoietic cells in anti-tumor immunity; adoptive transfer of WT NK cells into $\mathrm{NKLAM}^{-/-}$mice would confirm the role of $\mathrm{NKLAM}^{+} \mathrm{NK}$ cells in controlling tumor spread.

\section{NKLAM Function in Macrophages}

Macrophages are an important cellular component of the innate and adaptive immune systems and represent a first line of defense against invading pathogens. Macrophages employ oxidative and non-oxidative killing mechanisms to rid the host of pathogens. Since NKLAM is highly expressed in macrophages, we performed macrophage bacterial killing studies to determine whether NKLAM plays a role in macrophage bactericidal activity.

Transient transfection of J774 cells with NKLAM results in increased intracellular killing of E. coli compared with controltransfected cells. These results suggest that NKLAM enhances macrophage-mediated bacterial degradation. These data were confirmed in experiments using WT and NKLAM ${ }^{-/-}$BMDM and peritoneal macrophages isolated from WT and $\mathrm{NKLAM}^{-/-}$ mice; WT macrophages have greater bacterial killing activity than $\mathrm{NKLAM}^{-/-}$macrophages (Lawrence and Kornbluth, 2012).

We evaluated each of the steps associated with macrophage bactericidal activity. We measured phagocytosis of fluorescently labeled $E$. coli by flow cytometry and found no significant difference in uptake of bacteria between WT and $\mathrm{NKLAM}^{-/-}$ macrophages. We also assayed $\mathrm{pH}$ reduction during phagosome 
maturation by incubating macrophages with $E$. coli labeled with a $\mathrm{pH}$ sensitive fluorescent dye ( $\mathrm{pHrodo}$ ). There were no significant differences in phagosome $\mathrm{pH}$ between $\mathrm{WT}$ and $\mathrm{NKLAM}^{-/-}$ macrophages. Additionally, cleavage of cathepsin D, that occurs during phagosome maturation, is also similar between WT and NKLAM ${ }^{-/-}$macrophages (Lawrence and Kornbluth, 2012).

Phagosome maturation is a highly dynamic process. The number of proteins associated with the phagosome range from hundreds (Garin et al., 2001) to thousands (Trost et al., 2009). These proteins are involved in phagosome trafficking, protein degradation, phagosome acidification, and antigen presentation (Kinchen and Ravichandran, 2008). We isolated phagosomes from WT and NKLAM ${ }^{-/-}$macrophages and found that NKLAM is a component of macrophage phagosomes and co-localizes with phagosome proteins EEA-1 and LAMP-1 (Lawrence and Kornbluth, 2012). Using immunofluorescence, we localized NKLAM to the phagosome membrane surrounding ingested fluorescently labeled bacteria (Lawrence and Kornbluth, 2012). NKLAM expression in the phagosome is maximal at $\sim 35$ min post-ingestion and this correlates with increased ubiquitination of phagosome proteins; however, the identity of NKLAM phagosome targets is unknown. Ubiquitinated proteins on the phagosome membrane have been shown to interact with ESCRT (endosomal sorting complex required for transport) machinery and are necessary for protein sorting and phagosome maturation (Migliano and Teis, 2018). A recent study by Dean et al. (2019) suggests that post-translational modification of phagosome proteins (e.g., phosphorylation and ubiquitination) may transform the phagosome into a subcellular signaling platform. In support of this concept, a mass spectrometry (MS)based analysis of macrophage phagosome proteins revealed the presence of ubiquitin conjugation machinery including E1, E2, and E3 enzymes (Guo et al., 2015).

Since NKLAM is a membrane-bound protein, investigation of the orientation of the catalytic RING domain will determine whether NKLAM has access to phagosome cargo or cytosolic targets. If NKLAM is embedded in the phagosome membrane with its catalytic RING domains and C-terminal tail orientated into the cytoplasm, it would have access to phagosome membrane and cytoplasmic targets (Figure 4). In such an orientation, NKLAM would have access to bacteria that have evolved phagosomal escape mechanisms (e.g., Mycobacterium tuberculosis and Listeria monocytogenes). Another RBR ligase, Parkin, has been shown to ubiquitinate cytosolic M. tuberculosis, reducing its replication via destruction in the autophagolysosome (Manzanillo et al., 2013). Identification of NKLAM targets will be critical for determining the role of NKLAM in macrophage bactericidal function.

The processes of phagocytosis and autophagy have overlapping roles and mechanisms. LC3-associated phagocytosis (LAP) is a process in which some components of the autophagy pathway are recruited to the phagosome to lipidate LC3 molecules on a single membrane. Despite some overlap, LAP and canonical autophagy are distinct at the molecular, cellular, and functional levels. After phagocytic cargo uptake and phagosome formation, the class 3 PI-3-kinase complex (PI3KC3) is the first to be recruited to the membrane (Heckmann et al., 2017;
Herb et al., 2020). This process is shared between LAP and canonical autophagy. Affinity purification MS has identified numerous proteins that interact with NKLAM (Huttlin et al., 2015, 2017). The BioGRID ${ }^{2}$ database lists 56 interacting proteins; of these, three are members of the PI-3 kinase complex. This would place NKLAM not only in the phagosome membrane, but also in the membrane of the LAPosome. Our recent studies using a Sendai virus pneumonia model show that the lack of NKLAM negatively affects not only the conversion of LC3I to LC3II but the overall expression of LC3 (Lawrence et al., 2019). This observation implicates NKLAM as a potential regulator of LAP through its regulation of LC3 protein expression. Further studies are needed to determine whether NKLAM acts locally at the level of the LAPosome or more systemically at the transcriptional level.

\section{NKLAM Regulation of Immune-Associated Transcription Factor Activity}

Transcription factors that are crucial to the immune response are proteins of the STAT and NFКB families. Studies from our laboratory have shown that NKLAM plays an important role in regulating the activity of key members of both groups of transcription factors.

Using a transfection-based approach in HEK293 cells, we observed that NKLAM and STAT1 are associated in a protein complex (Lawrence and Kornbluth, 2016). Similar results were obtained using RAW 264.7 macrophages. In vitro, NKLAM is transiently localized to the interferon gamma receptor (IFNGR) during BMDM stimulation with IFN $\gamma$ (Lawrence and Kornbluth, 2016). STAT1 immunoprecipitated from WT and $\mathrm{NKLAM}^{-/-}$BMDM during IFN $\gamma$ stimulation shows evidence of increased transient K63-linked polyubiquitination in WT but not in $\mathrm{NKLAM}^{-/-}$cells. Total STAT1 levels are not significantly altered during the time course, suggesting that polyubiquitination does not induce large-scale STAT1 degradation (Lawrence and Kornbluth, 2016).

The observation that NKLAM associates with STAT1 and has a positive effect on its K63-linked polyubiquitination prompted us to determine if STAT1 DNA binding and/or transcriptional activity are affected by NKLAM. To that end, we performed oligonucleotide pull down assays. Our results demonstrated that the lack of NKLAM negatively affects STAT1 binding to an oligonucleotide containing a GAS (Lawrence and Kornbluth, 2016). In support of this observation, transfection studies using a GAS luciferase reporter plasmid demonstrated that STAT1mediated transcriptional activity is lower in $\mathrm{NKLAM}^{-/-}$than in WT cells (Lawrence and Kornbluth, 2016). Precisely which lysines of STAT1 serve as potential NKLAM ubiquitination targets and how ubiquitination affects STAT1 activity remain to be determined. Interestingly, a study by Huntelmann et al. (2014) determined that lysine 567 in STAT1 is required for GAS recognition; however, it has not been determined if that particular lysine is ubiquitinated. Recently, Guo et al. (2020) demonstrated

\footnotetext{
${ }^{2}$ https://thebiogrid.org/
} 

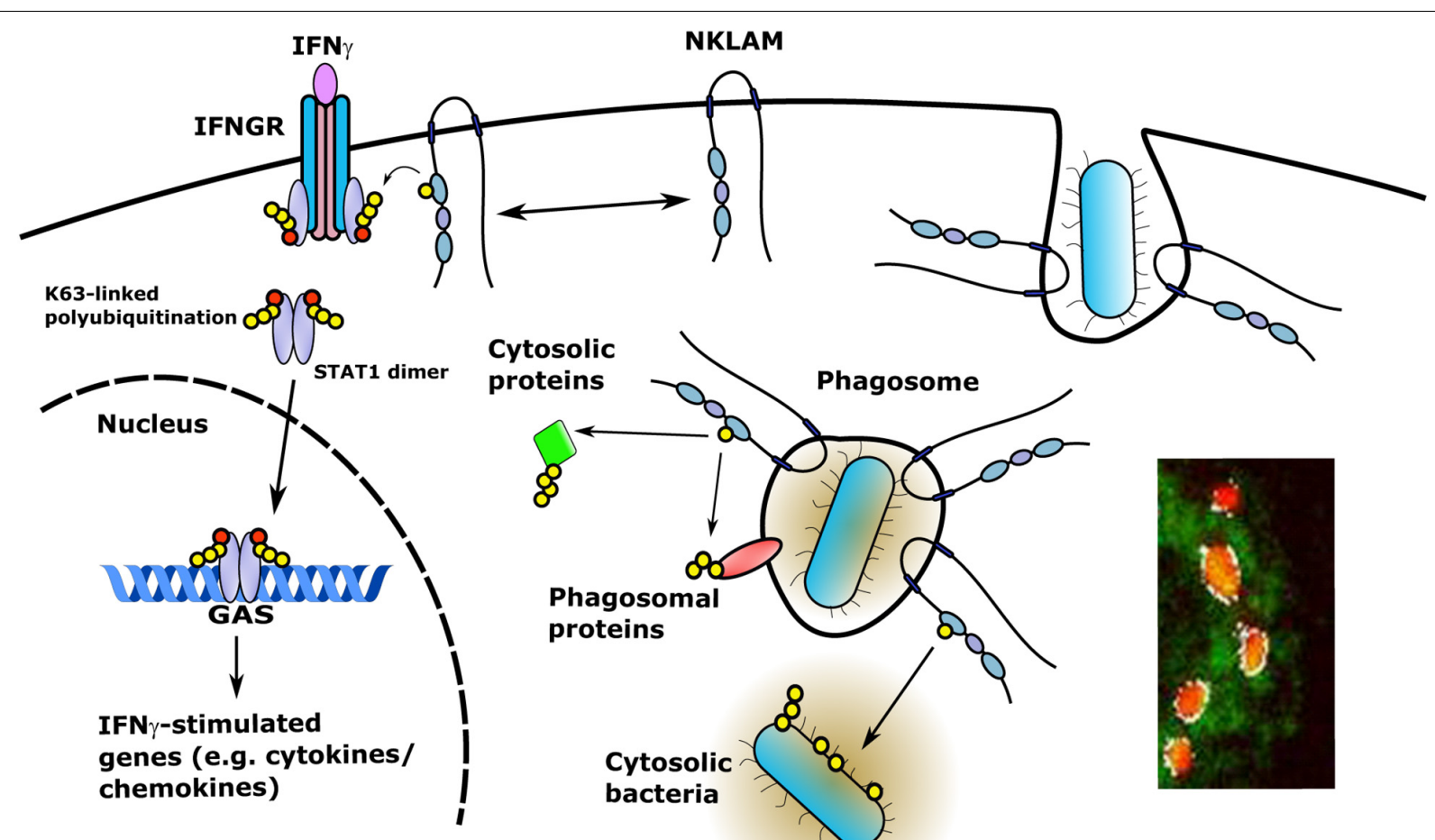

Cytosolic
proteins
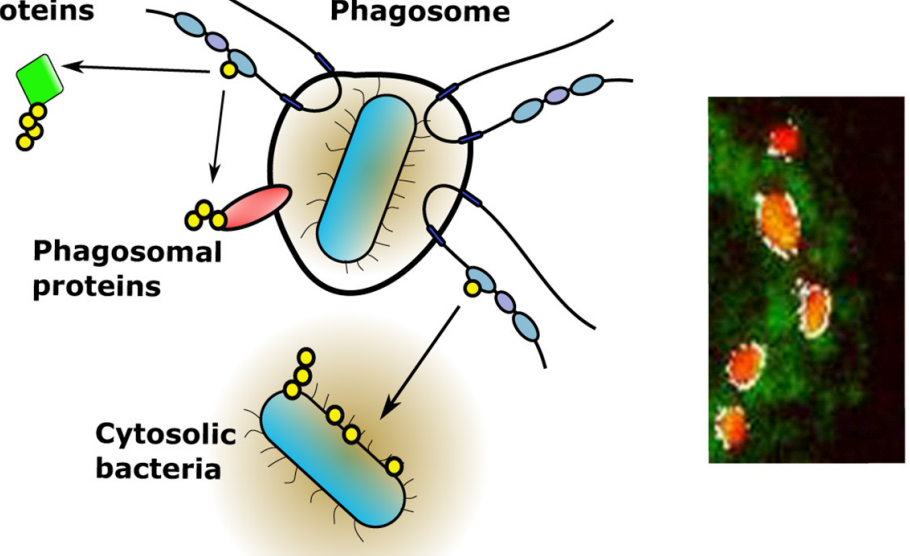

FIGURE 4 | NKLAM in macrophage immune function. Upon exposure to IFN $\gamma$, STAT1 is phosphorylated and NKLAM is transiently associated with the IFN $\gamma$-IFNGR complex at the plasma membrane. In this location, NKLAM is in close proximity to STAT1, which would allow NKLAM to ubiquitinate STAT1 or other proteins to promote STAT1 transcriptional activity. After ingestion of bacterial targets, NKLAM is localized to the phagosome membrane with the catalytic RING2 domain and C-terminal tail facing the cytoplasm. This would provide NKLAM with access to phagosome membrane proteins and cytosolic target proteins, as well as bacteria that escape the phagosome. Red circles; phosphotyrosine, yellow circles; ubiquitin. Immunomicrograph insert: Macrophage phagosomes after ingestion of fluorescent-labeled E. coli (red). NKLAM staining: green; Image J-defined co-localization of E. coli and NKLAM: white. Adapted from Lawrence, D.W., and Kornbluth, J. E3 ubiquitin ligase NKLAM is a macrophage phagosome protein and plays a role in bacterial killing. Copyright 2012, with permission from Elsevier and Lawrence D.W., and Kornbluth, J. E3 ubiquitin ligase NKLAM ubiquitinates STAT1 and positively regulates STAT1-mediated transcriptional activity. Copyright 2016, with permission from Elsevier.

that E3 ligase RNF220 mediated K63-linked ubiquitination of STAT1 at lysine 110 promotes the interaction between STAT1 and JAK1. Similar to our studies, the authors showed that RNF220 expression was induced by IFN $\gamma$ signaling and importantly, K63linked ubiquitination of STAT1 promoted cytokine induction (Guo et al., 2020). Additionally, others have shown that K63linked poly-ubiquitination of transcription factors is a positive regulator of transcriptional activity (Adhikary et al., 2005; Yao et al., 2018).

STAT1 phosphorylation precedes translocation into the nucleus and transcriptional activation. NKLAM affects the phosphorylation state of both STAT1 and STAT3 during active pulmonary infection. In a model of bacterial pneumonia, STAT1 and STAT3 phosphorylation in the lungs of mice infected with Streptococcus pneumoniae is significantly lower in $\mathrm{NKLAM}^{-/-}$ mice than in WT mice (Lawrence and Kornbluth, 2018). In support of this novel observation, we observed that phosphatase activity is significantly higher in $\mathrm{NKLAM}^{-/-}$mouse lungs infected with S. pneumoniae (Lawrence and Kornbluth, 2018). In a parallel study, using a model of viral pneumonia, STAT1 phosphorylation is also lower in lungs from $\mathrm{NKLAM}^{-/-}$ mice than from WT mice infected with Sendai virus (SeV) (Lawrence et al., 2019).

The NFкB pathway is considered the prototypical proinflammatory signaling cascade. NFKB regulates the expression of proinflammatory cytokines and chemokines crucial to the immune response. We found that nuclear translocation of the $\mathrm{NF \kappa B}$ protein p65 is significantly delayed in $\mathrm{NKLAM}^{-/-}$BMDM compared to WT macrophages after stimulation with LPS (Lawrence et al., 2015). The classical consensus nuclear localization signal (NLS) contains lysine and arginine residues (K-K/R-X-K/R), and is thus a potential target for ubiquitin ligases; however, data demonstrating that E3 ubiquitin ligases can positively regulate nuclear import via NLS ubiquitination is lacking.

Studies in macrophages treated with LPS have also shown that p65 phosphorylation at serine 536 is significantly attenuated in $\mathrm{NKLAM}^{-/-}$macrophages (Lawrence et al., 2015). Phosphorylation at serine 536 is associated with NFKB transcriptional activity (Giridharan and Srinivasan, 2018). In agreement with the above studies, $\mathrm{NKLAM}^{-/-}$macrophages have attenuated $\mathrm{NF} \kappa \mathrm{B}$ transcriptional activity as determined by 
a luciferase reporter assay, as well as lower expression of iNOS, a protein regulated by NFKB. Similarly, p65 phosphorylation in the lung is lower in NKLAM ${ }^{-/-}$mice than in WT mice infected with SeV (Lawrence et al., 2019).

Collectively, these data suggest that NKLAM is involved in regulating the phosphorylation state of two immunologically important transcription factors, and in doing so, positively modulates their transcriptional activity. An attractive hypothesis is that NKLAM promotes the ubiquitin-dependent degradation of key phosphatases, resulting in maintenance of the phosphorylated state of the transcription factor, thus sustaining its activation of gene expression. Studies are ongoing to test this hypothesis.

\section{Regulation of Cytokine and Chemokine Expression by NKLAM}

Cytokines and chemokines are immune regulators that control cell activation, migration, and differentiation. Many of the target genes of the STAT and NFKB family of transcription factors are cytokines and chemokines. This fact prompted our investigation into the regulation of cytokine and chemokine expression by NKLAM.

Early analyses of NK cells from $\mathrm{NKLAM}^{-/-}$mice found that they secrete significantly less IFN $\gamma$ than WT NK cells after target cell stimulation (Hoover et al., 2009). Initial in vitro studies demonstrated that BMDM and resident splenic macrophages from $\mathrm{NKLAM}^{-/-}$mice produce significantly less IFN $\beta$ than WT macrophages following treatment with LPS (Lawrence et al., 2015). Additionally, NKLAM ${ }^{-/-}$macrophages produce less IL-6, IFN $\gamma$, and MCP-1 than WT macrophages when treated with poly (I:C) (Lawrence et al., 2019). Tracheal epithelial cells isolated from $\mathrm{NKLAM}^{-/-}$mice produce significantly less IL6 and IFN $\gamma$ than WT cells when infected with SeV (Lawrence et al., 2019). Importantly, this observation demonstrates that the regulation of proinflammatory cytokine production by NKLAM is not limited to immune cells. In vivo infection studies confirm a role for NKLAM in regulating proinflammatory cytokine expression. Both viral (SeV) and bacterial (S. pneumoniae) pneumonia models demonstrate that the lack of NKLAM results in significantly lower cytokine/chemokine levels in the lungs and plasma of infected mice (Lawrence and Kornbluth, 2018; Lawrence et al., 2019).

There are several chemokines among the list of cytokines tested in our animal infection studies, including MCP-1, MIP-1 $\alpha$, RANTES, and KC (Table 1). These chemokines serve to recruit leukocytes to sites of inflammation. In infected $\mathrm{NKLAM}^{-/-}$ mice, chemokine expression is significantly lower than in WT mice. This corresponds to significantly fewer leukocytes recruited into the lungs of infected mice, as determined by both flow cytometric and histologic studies (Lawrence and Kornbluth, 2018; Lawrence et al., 2019). NKLAM ${ }^{-/-}$mice are not able to mount an immune response to $S$. pneumoniae and $\mathrm{SeV}$ comparable to WT mice.

Decreased cytokine and chemokine production in response to infection would suggest that $\mathrm{NKLAM}^{-/-}$mice are immunocompromised. Indeed, we found this to be the case when
TABLE 1 | Proinflammatory cytokines and chemokines were measured in $\mathrm{NKLAM}^{-/-}$and WT cells treated with LPS or IFN $\gamma$ in vitro and in biological tissues and organs from NKLAM ${ }^{-/-}$and WT mice exposed to live pathogens or TLR agonists in vivo.

\begin{tabular}{|c|c|c|c|}
\hline $\begin{array}{l}\text { Cytokine/ } \\
\text { Chemokine: }\end{array}$ & Stimulus: & Cell Type/Tissue: & References \\
\hline IL-1 & $\mathrm{SeV}$ & Lung & $\begin{array}{l}\text { Lawrence et al., } \\
2019\end{array}$ \\
\hline IL-2 & $\mathrm{SeV}$ & Lung & $\begin{array}{l}\text { Lawrence et al., } \\
2019\end{array}$ \\
\hline IL-6 & SeV, Poly (I:C) & $\begin{array}{l}\text { Lung, Tracheal } \\
\text { epithelial cells, } \\
\text { Macrophages } \\
\text { (BMDM) }\end{array}$ & $\begin{array}{l}\text { Lawrence et al., } \\
2019\end{array}$ \\
\hline IL-12 & $\begin{array}{l}\text { SeV, } \\
\text { S. pneumoniae }\end{array}$ & Lung & $\begin{array}{l}\text { Lawrence et al., } \\
2019 \text {; } \\
\text { Lawrence and } \\
\text { Kornbluth, } \\
2018\end{array}$ \\
\hline IL-17 & $\mathrm{SeV}$ & Lung & $\begin{array}{l}\text { Lawrence et al., } \\
2019\end{array}$ \\
\hline $\mathrm{IFN} \gamma$ & $\begin{array}{l}\text { Tumor cell } \\
\text { stimulation, SeV, } \\
\text { S. pneumoniae, } \\
\text { Poly (I:C) }\end{array}$ & $\begin{array}{l}\text { NK cells, Lung, } \\
\text { Plasma, } \\
\text { Macrophages } \\
\text { (BMDM) }\end{array}$ & $\begin{array}{l}\text { Hoover et al., } \\
\text { 2009; } \\
\text { Lawrence and } \\
\text { Kornbluth, } \\
\text { 2018; } \\
\text { Lawrence et al., } \\
2019\end{array}$ \\
\hline IFN $\beta$ & LPS, SeV & $\begin{array}{l}\text { Macrophages } \\
\text { (BMDM), } \\
\text { Macrophages } \\
\text { (spleen), Tracheal } \\
\text { epithelial cells }\end{array}$ & $\begin{array}{l}\text { Lawrence et al., } \\
2015 \text {; } \\
\text { Lawrence et al., } \\
2019\end{array}$ \\
\hline $\mathrm{TNF} \alpha$ & $\begin{array}{l}\text { SeV, } \\
\text { S. pneumoniae }\end{array}$ & Lung & $\begin{array}{l}\text { Lawrence et al., } \\
2019 \text {; } \\
\text { Lawrence and } \\
\text { Kornbluth, } \\
2018\end{array}$ \\
\hline $\mathrm{KC}$ & $\mathrm{SeV}$ & Lung & $\begin{array}{l}\text { Lawrence et al., } \\
2019\end{array}$ \\
\hline MCP-1 & $\begin{array}{l}\text { SeV, } \\
\text { S. pneumoniae, } \\
\text { Poly (l:C) }\end{array}$ & $\begin{array}{l}\text { Lung, Plasma, } \\
\text { Macrophages } \\
\text { (BMDM) }\end{array}$ & $\begin{array}{l}\text { Lawrence and } \\
\text { Kornbluth, } \\
\text { 2018; } \\
\text { Lawrence et al., } \\
2019\end{array}$ \\
\hline RANTES & $\mathrm{IFN} \gamma, \mathrm{SeV}$ & $\begin{array}{l}\text { Macrophages } \\
\text { (BMDM), Lung }\end{array}$ & $\begin{array}{l}\text { Lawrence and } \\
\text { Kornbluth, } \\
\text { 2016; } \\
\text { Lawrence et al., } \\
2019\end{array}$ \\
\hline $\mathrm{MIP}-1 \alpha$ & $\mathrm{SeV}$ & Lung & $\begin{array}{l}\text { Lawrence et al., } \\
2019\end{array}$ \\
\hline $\mathrm{MIP}-1 \beta$ & $\mathrm{SeV}$ & Lung & $\begin{array}{l}\text { Lawrence et al., } \\
2019\end{array}$ \\
\hline MIP-2 & $\mathrm{SeV}$ & Lung & $\begin{array}{l}\text { Lawrence et al., } \\
2019\end{array}$ \\
\hline GM-CSF & $\mathrm{SeV}$ & Lung & $\begin{array}{l}\text { Lawrence et al., } \\
2019\end{array}$ \\
\hline G-CSF & $\mathrm{SeV}$ & Lung & $\begin{array}{l}\text { Lawrence et al., } \\
2019\end{array}$ \\
\hline $\mid \mathrm{P}-10$ & $\mathrm{SeV}$ & Lung & $\begin{array}{l}\text { Lawrence et al., } \\
2019\end{array}$ \\
\hline Eotaxin-1 & $\mathrm{SeV}$ & Lung & $\begin{array}{l}\text { Lawrence et al., } \\
2019\end{array}$ \\
\hline$A X L$ & $\mathrm{SeV}$ & Lung & $\begin{array}{l}\text { Lawrence et al., } \\
2019\end{array}$ \\
\hline
\end{tabular}


we infected mice with a large dose of Sendai virus. Eighty percent of $\mathrm{NKLAM}^{-/-}$mice died 8 days post-infection compared to only $20 \%$ of WT mice. A large, pathogenic challenge overwhelms $\mathrm{NKLAM}^{-/-}$mice and leads to rapid mortality. The lack of a robust immune response by $\mathrm{NKLAM}^{-/-}$mice in this instance is detrimental. However, when smaller doses of infectious agents (either $\mathrm{SeV}$ or S. pneumoniae) are given, $\mathrm{NKLAM}^{-/-}$mice are afforded a slight survival benefit. This is likely due to less inflammatory leukocytes in the lungs of $\mathrm{NKLAM}^{-/-}$mice, which would correspond to less host-mediated tissue destruction during inflammation.

These data suggest a model for a role of NKLAM in STAT1mediated transcriptional activity in macrophages, in cytokine and chemokine production and in their phagosome-mediated pathogen destruction (Figure 4$)^{3}$. Overall, these studies are defining NKLAM as a key component of the innate immune system. Further research into NKLAM substrates and potential immune signaling pathways is warranted to identify novel control points for the therapeutic modulation of inflammation.

\section{Links Between RBR Family Members and Immune Functions}

There is very limited published data from other investigators regarding the expression/function of NKLAM. However, they collectively point to a role of NKLAM in innate immunity and response to infectious agents. One report showed that NKLAM expression is increased in chickens infected with pathogenic avian influenza virus; this increase is associated with survivability (Uchida et al., 2012). Another study documented upregulation of NKLAM expression in salmon exposed to infectious salmon anemia virus (Li et al., 2011). NKLAM levels are also significantly increased in grass carp infected with grass carp reovirus (Luo et al., 2019). The precise function of NKLAM in these infection models was not determined, but implicates NKLAM as an important component of innate immunity.

A study comparing gene expression in monocytes from adults with low versus high peak bone mass was performed to identify potential genes associated with osteoclast differentiation (Xiao et al., 2012). One of the candidate genes was NKLAM, suggesting that it plays a role in osteoporosis. NKLAM (RNF19B) gene expression was also upregulated, along with other proinflammatory genes, in the peripheral blood of Chinese patients with acute myocardial infarction compared to healthy controls (Su et al., 2018). Survey of the Gene Expression Omnibus $(\mathrm{GEO})^{4}$ of microarray analyses identified elevation of NKLAM mRNA expression in monocytes and macrophages exposed to LPS, Borrelia burgdorferi (the spirochete responsible for Lyme disease), Chlamydia pneumoniae, Francisella tularensis (the causative agent for tularemia), Porphyromonas gingivalis (the pathogenic bacterium associated with periodontitis),

\footnotetext{
${ }^{3}$ Adapted from Lawrence, D.W., and Kornbluth, J. E3 ubiquitin ligase NKLAM is a macrophage phagosome protein and plays a role in bacterial killing. Copyright 2012, with permission from Elsevier and Lawrence D.W., and Kornbluth, J. E3 ubiquitin ligase NKLAM ubiquitinates STAT1 and positively regulates STAT1mediated transcriptional activity. Copyright 2016, with permission from Elsevier. ${ }^{4}$ https://www.ncbi.nlm.nih.gov/geo/
}

M. tuberculosis, as well as upon infection with several adenoviruses and rhinoviruses that cause respiratory infections.

Other RBR family members have also been implicated in innate immune function. Alterations in the Parkin gene (PARK2) have been associated with increased susceptibility to Salmonella typhi, Salmonella paratyphi (Ali et al., 2006), and Mycobacterium leprae (Mira et al., 2004; Malhotra et al., 2006). Additionally, Parkin-deficient mice and Drosophila melanogaster are highly susceptible to infection with $M$. tuberculosis (Manzanillo et al., 2013). In a recent study, Aalto et al. (2019) found that RBR family member HOIP (HOIL-1L interacting protein) ortholog LUBEL was required for $D$. melanogaster to survive oral challenge with Gram-negative bacteria.

\section{Role of NKLAM in Non-Immune Functions}

Hematopoietic cell activation is not the only circumstance that elicits NKLAM expression. In searching for ubiquitin ligases that may be involved in protein degradation within the endoplasmic reticulum (ER), Kaneko et al. (2016) found that HeLa cells undergoing ER stress, caused by exposure to thapsigargin or tunicamycin, upregulated NKLAM mRNA expression. In another study, it was found that MCF7 breast cancer cells upregulate NKLAM expression when exposed to oxyphenisatin acetate, a drug that was being tested as a potential cancer therapeutic (Morrison et al., 2013). This drug is known to have antiproliferative activity, but the investigators learned that treatment with this drug is also associated with autophagy, mitochondrial dysfunction, and generation of reactive oxygen species.

NKLAM mRNA expression is induced in mouse tracheal epithelial cells infected with SeV (Lawrence et al., 2019). However, these levels are extremely low compared to that seen in NK cells and macrophages. The role of non-hematopoietic cells in the phenotype we observe in $\mathrm{NKLAM}^{-/-}$mice is uncertain. Reconstitution of these mice with WT bone marrow will help answer this question.

Protein ubiquitination is a critical regulatory mechanism of autophagy, controlling its initiation, execution, and termination (Chen et al., 2019). One of our recent studies suggests that NKLAM may play a role in autophagic flux. Upon induction of autophagy by treatment with rapamycin, $\mathrm{NKLAM}^{-/-}$ macrophages convert less LC3I to LC3II and translocate less LC3 to autophagosomes than WT macrophages. These indicators of autophagy suggest that NKLAM is associated with autophagic flux and expression of key autophagy-related proteins (Lawrence et al., 2019). Further studies are underway to analyze the role of NKLAM in autophagy.

\section{FUTURE DIRECTIONS}

Identification of the role of the ubiquitin ligase activity of NKLAM in vitro and in vivo is of paramount importance. The derivation of an NKLAM ${ }^{-/-}$NK3.3 cell line is in development to further delineate the role NKLAM plays in human NK cell effector function. This cell line, in combination with fluorescently 
tagged NKLAM WT and ligase-inactive constructs, will provide the ability to monitor and measure differences between WT and NKLAM ${ }^{-/-} \mathrm{NK}$ cells during activation, effector function, and target cell death. Similarly, these NKLAM constructs will allow us to track NKLAM in macrophages during the process of phagocytosis and pathogen destruction and facilitate identification of NKLAM-interacting proteins during this event. Comparison of cells expressing WT and NKLAM constructs with mutations in the RBR domain (C302A) will be critical for establishing the role of ubiquitination in the function of NKLAM. Similarly, introduction of WT and catalytically inactive NKLAM into NKLAM ${ }^{-/-}$mice would allow for studies of the ubiquitination function of NKLAM in vivo.

Experimental evidence that defines specific targets of RBR ligases is limited. The exception is RBR ligase Parkin, which has been shown to ubiquitinate dozens of proteins (Sarraf et al., 2013). The identification of NKLAM substrates would provide insight into the cellular mechanisms regulated by NKLAMmediated ubiquitination. To this end, the use of diGly capture and MS detection methodologies followed by bioinformatic comparison of WT and NKLAM ${ }^{-/-}$ubiquitomes will allow us to define specific NKLAM target proteins. Identification of NKLAM substrates, as well as the specific lysines targeted by NKLAM, will allow for the development of reagents that can be used to modulate NKLAM function in the context of innate immunity.

Several proteins that interact with NKLAM have been identified by affinity purification MS; 56 in the BioGRID database and 71 in IntAct $^{5}$. The biological/functional validity and significance of these protein associations remains to be determined.

The prediction that intrinsically disordered domains may bind RNA or DNA provides an interesting direction for research on NKLAM. RNA-protein interactions can be divided into two categories: (1) RNA acting on the RNA binding protein (RBP) (Lunde et al., 2007) or (2) the RBP acting on the RNA (Oliveira et al., 2017). When RNA acts on the RBP, it can lead to changes in RBP function, localization, stability, or interactions. An RBP acting on the RNA can change RNA stability, processing or modification. DNA binding proteins would be localized to the nucleus and likely play a role in gene transcription, replication, repair or the regulation of those processes.

The potential for these disordered regions to interact with a variety of proteins and for these interactions to be controlled by post-translational modifications will make studying these interactions inherently difficult. NKLAM expression is

${ }^{5}$ https://www.ebi.ac.uk/intact/

\section{REFERENCES}

Aalto, A. L., Mohan, A. K., Schwintzer, L., Kupka, S., Kietz, C., Walczak, H., et al. (2019). M1-linked ubiquitination by LUBEL is required for inflammatory responses to oral infection in Drosophila. Cell Death Differ. 26, 860-876. doi: 10.1038/s41418-018-0164-x

Adhikary, S., Marinoni, F., Hock, A., Hulleman, E., Popov, N., Beier, R., et al. (2005). The ubiquitin ligase HectH9 regulates transcriptional activation by Myc tightly regulated and induced under specific activation conditions. These conditions may alter the post-translational modifications of NKLAM, thereby exposing it to different combinations of interactors (protein, DNA, and RNA). It will be necessary to not just identify which region an interacting protein binds to but also under what conditions it interacts with NKLAM, as well as differentiate between interacting proteins and ubiquitinated substrates.

Studies to date have shown that NKLAM is a unique and important ubiquitin ligase that participates in multiple regulatory pathways essential for immune function. A greater understanding of its role in controlling infection, inflammation and antitumor activity will hopefully lead to the development of new strategies for treating bacterial and viral infections, sepsis, autoimmunity, and cancer.

\section{AUTHOR CONTRIBUTIONS}

DL, PW, AC, EM, and JK contributed to the writing and editing of this review article. DL prepared the figures and table. The research performed by the Kornbluth laboratory was funded by grants obtained by JK. All authors are accountable for the content of this review article.

\section{FUNDING}

This research by the Kornbluth laboratory described in this review article was funded by Merit Awards (I01 BX000705) provided to JK by the United States Department of Veterans Affairs, Biomedical Laboratory Research and Development Service $^{6}$. The funders had no role in study design, data collection and analysis, decision to publish, or preparation of the manuscript. Additional funding was provided by the Department of Pathology and Saint Louis University.

\section{ACKNOWLEDGMENTS}

The authors thank the students, fellows and staff who contributed to the studies of NKLAM over the past 20 years. The authors also thank the Research Microscopy Core, Flow Cytometry Core, and Comparative Medicine staff at Saint Louis University for their expertise and support.

\footnotetext{
${ }^{6}$ https://www.va.gov/
}

and is essential for tumor cell proliferation. Cell 123, 409-421. doi: 10.1016/j. cell.2005.08.016

Ali, S., Vollaard, A. M., Widjaja, S., Surjadi, C., Van De Vosse, E., and Van Dissel, J. T. (2006). PARK2/PACRG polymorphisms and susceptibility to typhoid and paratyphoid fever. Clin. Exp. Immunol. 144, 425-431. doi: 10.1111/j.1365-2249. 2006.03087.x

Ambrose, E. C., and Kornbluth, J. (2009). Downregulation of uridine-cytidine kinase like-1 decreases proliferation and enhances tumor susceptibility to lysis 
by apoptotic agents and natural killer cells. Apoptosis 14, 1227-1236. doi: 10.1007/s10495-009-0385-z

Barik, A., Katuwawala, A., Hanson, J., Paliwal, K., Zhou, Y., and Kurgan, L. (2020). DEPICTER: intrinsic disorder and disorder function prediction server. J. Mol. Biol. 432, 3379-3387. doi: 10.1016/j.jmb.2019.12.030

Burkhardt, J. K., Hester, S., Lapham, C. K., and Argon, Y. (1990). The lytic granules of natural killer cells are dual-function organelles combining secretory and prelysosomal compartments. J. Cell Biol. 111, 2327-2340. doi: 10.1083/jcb.111.6. 2327

Cerwenka, A., Baron, J. L., and Lanier, L. L. (2001). Ectopic expression of retinoic acid early inducible-1 gene (RAE-1) permits natural killer cell-mediated rejection of a MHC class I-bearing tumor in vivo. Proc. Natl. Acad. Sci. U.S.A. 98, 11521-11526. doi: 10.1073/pnas.201238598

Chen, R. H., Chen, Y. H., and Huang, T. Y. (2019). Ubiquitin-mediated regulation of autophagy. J. Biomed. Sci. 26:80. doi: 10.1186/s12929-019-0569-y

Cheng, W. S., Tao, H., Hu, E. P., Liu, S., Cai, H. R., Tao, X. L., et al. (2014). Both genes and lncRNAs can be used as biomarkers of prostate cancer by using high throughput sequencing data. Eur. Rev. Med. Pharmacol. Sci. 18, 3504-3510.

Darling, A. L., and Uversky, V. N. (2018). Intrinsic disorder and posttranslational modifications: the darker side of the biological dark matter. Front. Genet. 9:158. doi: 10.3389/fgene.2018.00158

De Saint Basile, G., Menasche, G., and Fischer, A. (2010). Molecular mechanisms of biogenesis and exocytosis of cytotoxic granules. Nat. Rev. Immunol. 10, 568-579. doi: 10.1038/nri2803

Dean, P., Heunis, T., Hartlova, A., and Trost, M. (2019). Regulation of phagosome functions by post-translational modifications: a new paradigm. Curr. Opin. Chem. Biol. 48, 73-80. doi: 10.1016/j.cbpa.2018.11.001

Diefenbach, A., Jensen, E. R., Jamieson, A. M., and Raulet, D. H. (2001). Rae1 and H60 ligands of the NKG2D receptor stimulate tumour immunity. Nature 413 , 165-171. doi: 10.1038/35093109

Dove, K. K., and Klevit, R. E. (2017). RING-between-RING E3 ligases: emerging themes amid the variations. J. Mol. Biol. 429, 3363-3375. doi: 10.1016/j.jmb. 2017.08.008

Dunker, A. K., Cortese, M. S., Romero, P., Iakoucheva, L. M., and Uversky, V. N. (2005). Flexible nets. The roles of intrinsic disorder in protein interaction networks. FEBS J. 272, 5129-5148. doi: 10.1111/j.1742-4658.2005.04948.x

Dyson, H. J. (2016). Making sense of intrinsically disordered proteins. Biophys. J. 110, 1013-1016. doi: 10.1016/j.bpj.2016.01.030

Ewens, A., Mihich, E., and Ehrke, M. J. (2005). Distant metastasis from subcutaneously grown E0771 medullary breast adenocarcinoma. Anticancer Res. 25, 3905-3915.

Fardilha, M., Esteves, S. L., Korrodi-Gregorio, L., Vintem, A. P., Domingues, S. C., Rebelo, S., et al. (2011). Identification of the human testis protein phosphatase 1 interactome. Biochem. Pharmacol. 82, 1403-1415. doi: 10.1016/j.bcp.2011.02. 018

Fortier, J. M., and Kornbluth, J. (2006). NK lytic-associated molecule, involved in NK cytotoxic function, is an E3 ligase. J. Immunol. 176, 6454-6463. doi: 10.4049/jimmunol.176.11.6454

Garin, J., Diez, R., Kieffer, S., Dermine, J. F., Duclos, S., Gagnon, E., et al. (2001). The phagosome proteome: insight into phagosome functions. J. Cell Biol. 152, 165-180. doi: $10.1083 /$ jcb.152.1.165

Geiger, T., Madden, S. F., Gallagher, W. M., Cox, J., and Mann, M. (2012). Proteomic portrait of human breast cancer progression identifies novel prognostic markers. Cancer Res. 72, 2428-2439. doi: 10.1158/0008-5472.CAN11-3711

Giridharan, S., and Srinivasan, M. (2018). Mechanisms of NF-kappaB p65 and strategies for therapeutic manipulation. J. Inflamm. Res. 11, 407-419. doi: 10.2147/JIR.S140188

Gorelik, E., Wiltrout, R. H., Okumura, K., Habu, S., and Herberman, R. B. (1982). Role of NK cells in the control of metastatic spread and growth of tumor cells in mice. Int. J. Cancer 30, 107-112. doi: 10.1002/ijc.2910300118

Gu, J. W., Young, E., Busby, B., Covington, J., and Johnson, J. W. (2009). Oral administration of pyrrolidine dithiocarbamate (PDTC) inhibits VEGF expression, tumor angiogenesis, and growth of breast cancer in female mice. Cancer Biol. Ther. 8, 514-521. doi: 10.4161/cbt.8.6.7689

Gullickson, G., Ambrose, E. C., Hoover, R. G., and Kornbluth, J. (2016). Uridine cytidine kinase like-1 enhances tumor cell proliferation and mediates protection from natural killer-mediated killing. Int. J. Immunol. Immunother. 3. doi: 10.23937/2378-3672/1410018

Guo, M., Hartlova, A., Dill, B. D., Prescott, A. R., Gierlinski, M., and Trost, M. (2015). High-resolution quantitative proteome analysis reveals substantial differences between phagosomes of RAW 264.7 and bone marrow derived macrophages. Proteomics 15, 3169-3174. doi: 10.1002/pmic.201400431

Guo, X., Ma, P., Li, Y., Yang, Y., Wang, C., Xu, T., et al. (2020). RNF220 mediates K63-linked polyubiquitination of STAT1 and promotes host defense. Cell Death Differ. doi: 10.1038/s41418-020-00609-7 [Epub ahead of print].

Hanna, N., and Burton, R. C. (1981). Definitive evidence that natural killer (NK) cells inhibit experimental tumor metastases in vivo. J. Immunol. 127, 1754-1758.

Heckmann, B. L., Boada-Romero, E., Cunha, L. D., Magne, J., and Green, D. R. (2017). LC3-associated phagocytosis and inflammation. J. Mol. Biol. 429, 35613576. doi: 10.1016/j.jmb.2017.08.012

Herb, M., Gluschko, A., and Schramm, M. (2020). LC3-associated phagocytosis - the highway to hell for phagocytosed microbes. Semin. Cell Dev. Biol. 101, 68-76. doi: 10.1016/j.semcdb.2019.04.016

Hochstrasser, M. (1996). Ubiquitin-dependent protein degradation. Annu. Rev. Genet. 30, 405-439. doi: 10.1146/annurev.genet.30.1.405

Hoover, R. G., Gullickson, G., and Kornbluth, J. (2009). Impaired NK cytolytic activity and enhanced tumor growth in NK lytic-associated molecule-deficient mice. J. Immunol. 183, 6913-6921. doi: 10.4049/jimmunol.0901679

Hoover, R. G., Gullickson, G., and Kornbluth, J. (2012). Natural killer lyticassociated molecule plays a role in controlling tumor dissemination and metastasis. Front. Immunol. 3:393. doi: 10.3389/fimmu.2012.00393

Huntelmann, B., Staab, J., Herrmann-Lingen, C., and Meyer, T. (2014). A conserved motif in the linker domain of STAT1 transcription factor is required for both recognition and release from high-affinity DNA-binding sites. PLoS One 9:e97633. doi: 10.1371/journal.pone.0097633

Huttlin, E. L., Bruckner, R. J., Paulo, J. A., Cannon, J. R., Ting, L., Baltier, K., et al. (2017). Architecture of the human interactome defines protein communities and disease networks. Nature 545, 505-509. doi: 10.1038/nature22366

Huttlin, E. L., Ting, L., Bruckner, R. J., Gebreab, F., Gygi, M. P., Szpyt, J., et al. (2015). The BioPlex network: a systematic exploration of the human interactome. Cell 162, 425-440. doi: 10.1016/j.cell.2015.06.043

Jong, A. Y., Wu, C. H., Li, J., Sun, J., Fabbri, M., Wayne, A. S., et al. (2017) Large-scale isolation and cytotoxicity of extracellular vesicles derived from activated human natural killer cells. J. Extracell. Vesicles 6:1294368. doi: 10. 1080/20013078.2017.1294368

Kaneko, M., Iwase, I., Yamasaki, Y., Takai, T., Wu, Y., Kanemoto, S., et al. (2016) Genome-wide identification and gene expression profiling of ubiquitin ligases for endoplasmic reticulum protein degradation. Sci. Rep. 6:30955. doi: 10.1038/ srep30955

Kawasaki, T., and Kawai, T. (2014). Toll-like receptor signaling pathways. Front. Immunol. 5:461. doi: 10.3389/fimmu.2014.00461

Kim, S., Iizuka, K., Aguila, H. L., Weissman, I. L., and Yokoyama, W. M. (2000). In vivo natural killer cell activities revealed by natural killer cell-deficient mice. Proc. Natl. Acad. Sci. U.S.A. 97, 2731-2736. doi: 10.1073/pnas.050588297

Kinchen, J. M., and Ravichandran, K. S. (2008). Phagosome maturation: going through the acid test. Nat. Rev. Mol. Cell Biol. 9, 781-795. doi: 10.1038/nrm2515

Kitada, T., Asakawa, S., Hattori, N., Matsumine, H., Yamamura, Y., Minoshima, S., et al. (1998). Mutations in the parkin gene cause autosomal recessive juvenile parkinsonism. Nature 392, 605-608. doi: 10.1038/33416

Kornbluth, J., Flomenberg, N., and Dupont, B. (1982). Cell surface phenotype of a cloned line of human natural killer cells. J. Immunol. 129, 2831-2837.

Kornbluth, J., and Hoover, R. G. (1988). Changes in gene expression associated with IFN-beta and IL-2-induced augmentation of human natural killer cell function. J. Immunol. 141, 3234-3240.

Kozlowski, M., Schorey, J., Portis, T., Grigoriev, V., and Kornbluth, J. (1999). NK lytic-associated molecule: a novel gene selectively expressed in cells with cytolytic function. J. Immunol. 163, 1775-1785.

Lawrence, D. W., Gullickson, G., and Kornbluth, J. (2015). E3 ubiquitin ligase NKLAM positively regulates macrophage inducible nitric oxide synthase expression. Immunobiology 220, 83-92. doi: 10.1016/j.imbio.2014.08.016

Lawrence, D. W., and Kornbluth, J. (2012). E3 ubiquitin ligase NKLAM is a macrophage phagosome protein and plays a role in bacterial killing. Cell. Immunol. 279, 46-52. doi: 10.1016/j.cellimm.2012.09.004 
Lawrence, D. W., and Kornbluth, J. (2016). E3 ubiquitin ligase NKLAM ubiquitinates STAT1 and positively regulates STAT1-mediated transcriptional activity. Cell. Signal. 28, 1833-1841. doi: 10.1016/j.cellsig.2016.08.014

Lawrence, D. W., and Kornbluth, J. (2018). Reduced inflammation and cytokine production in NKLAM deficient mice during Streptococcus pneumoniae infection. PLoS One 13:e0194202. doi: 10.1371/journal.pone.0194202

Lawrence, D. W., Shornick, L. P., and Kornbluth, J. (2019). Mice deficient in NKLAM have attenuated inflammatory cytokine production in a Sendai virus pneumonia model. PLoS One 14:e0222802. doi: 10.1371/journal.pone.0222802

Lechtenberg, B. C., Rajput, A., Sanishvili, R., Dobaczewska, M. K., Ware, C. F., Mace, P. D., et al. (2016). Structure of a HOIP/E2 ubiquitin complex reveals RBR E3 ligase mechanism and regulation. Nature 529, 546-550. doi: 10.1038/ nature 16511

Li, J., Boroevich, K. A., Koop, B. F., and Davidson, W. S. (2011). Comparative genomics identifies candidate genes for infectious salmon anemia (ISA) resistance in Atlantic salmon (Salmo salar). Mar. Biotechnol. 13, 232-241. doi: 10.1007/s10126-010-9284-0

Lugini, L., Cecchetti, S., Huber, V., Luciani, F., Macchia, G., Spadaro, F., et al. (2012). Immune surveillance properties of human NK cell-derived exosomes. J. Immunol. 189, 2833-2842. doi: 10.4049/jimmunol.1101988

Lunde, B. M., Moore, C., and Varani, G. (2007). RNA-binding proteins: modular design for efficient function. Nat. Rev. Mol. Cell Biol. 8, 479-490. doi: 10.1038/ nrm 2178

Luo, L., Zhu, D., Huang, R., Xiong, L., Mehjabin, R., He, L., et al. (2019). Molecular cloning and preliminary functional analysis of six RING-between-ring (RBR) genes in grass carp (Ctenopharyngodon idellus). Fish Shellfish Immunol. 87, 62-72. doi: 10.1016/j.fsi.2018.12.078

Malhotra, D., Darvishi, K., Lohra, M., Kumar, H., Grover, C., Sood, S., et al. (2006). Association study of major risk single nucleotide polymorphisms in the common regulatory region of PARK2 and PACRG genes with leprosy in an Indian population. Eur. J. Hum. Genet. 14, 438-442. doi: 10.1038/sj.ejhg. 5201563

Manzanillo, P. S., Ayres, J. S., Watson, R. O., Collins, A. C., Souza, G., Rae, C. S., et al. (2013). The ubiquitin ligase parkin mediates resistance to intracellular pathogens. Nature 501,512-516. doi: 10.1038/nature12566

Martino, L., Brown, N. R., Masino, L., Esposito, D., and Rittinger, K. (2018). Determinants of E2-ubiquitin conjugate recognition by RBR E3 ligases. Sci. Rep. 8:68. doi: 10.1038/s41598-017-18513-5

Metzger, M. B., Pruneda, J. N., Klevit, R. E., and Weissman, A. M. (2014). RINGtype E3 ligases: master manipulators of E2 ubiquitin-conjugating enzymes and ubiquitination. Biochim. Biophys. Acta 1843, 47-60. doi: 10.1016/j.bbamcr. 2013.05.026

Migliano, S. M., and Teis, D. (2018). ESCRT and membrane protein ubiquitination. Prog. Mol. Subcell. Biol. 57, 107-135. doi: 10.1007/978-3-319-96704-2_4

Mira, M. T., Alcais, A., Nguyen, V. T., Moraes, M. O., Di Flumeri, C., Vu, H. T., et al. (2004). Susceptibility to leprosy is associated with PARK2 and PACRG. Nature 427, 636-640. doi: 10.1038/nature02326

Morrison, B. L., Mullendore, M. E., Stockwin, L. H., Borgel, S., Hollingshead, M. G., and Newton, D. L. (2013). Oxyphenisatin acetate (NSC 59687) triggers a cell starvation response leading to autophagy, mitochondrial dysfunction, and autocrine TNFalpha-mediated apoptosis. Cancer Med. 2, 687-700. doi: 10.1002/cam4.107

Oliveira, C., Faoro, H., Alves, L. R., and Goldenberg, S. (2017). RNA-binding proteins and their role in the regulation of gene expression in Trypanosoma cruzi and Saccharomyces cerevisiae. Genet. Mol. Biol. 40, 22-30. doi: 10.1590/ 1678-4685-gmb-2016-0258

Owen, I., and Shewmaker, F. (2019). The role of post-translational modifications in the phase transitions of intrinsically disordered proteins. Int. J. Mol. Sci. 20:5501. doi: 10.3390/ijms20215501

Portis, T., Anderson, J., Esposito, A., and Kornbluth, J. (2000). Gene structure of human and mouse NKLAM, a gene associated with cellular cytotoxicity. Immunogenetics 51, 546-555. doi: 10.1007/s002510000182
Sarraf, S. A., Raman, M., Guarani-Pereira, V., Sowa, M. E., Huttlin, E. L., Gygi, S. P., et al. (2013). Landscape of the PARKIN-dependent ubiquitylome in response to mitochondrial depolarization. Nature 496, 372-376. doi: 10.1038/nature12043

Smit, J. J., and Sixma, T. K. (2014). RBR E3-ligases at work. EMBO Rep. 15, 142-154. doi: 10.1002/embr.201338166

Spratt, D. E., Walden, H., and Shaw, G. S. (2014). RBR E3 ubiquitin ligases: new structures, new insights, new questions. Biochem. J. 458, 421-437. doi: 10.1042/ BJ20140006

Su, J., Gao, C., Wang, R., Xiao, C., and Yang, M. (2018). Genes associated with inflammation and the cell cycle may serve as biomarkers for the diagnosis and prognosis of acute myocardial infarction in a Chinese population. Mol. Med. Rep. 18, 1311-1322. doi: 10.3892/mmr.2018.9077

Sun, Y. (2006). E3 ubiquitin ligases as cancer targets and biomarkers. Neoplasia 8, 645-654. doi: 10.1593/neo.06376

Swatek, K. N., and Komander, D. (2016). Ubiquitin modifications. Cell Res. 26, 399-422. doi: 10.1038/cr.2016.39

Trost, M., English, L., Lemieux, S., Courcelles, M., Desjardins, M., and Thibault, P. (2009). The phagosomal proteome in interferon-gammaactivated macrophages. Immunity 30, 143-154. doi: 10.1016/j.immuni.2008. 11.006

Uchida, Y., Watanabe, C., Takemae, N., Hayashi, T., Oka, T., Ito, T., et al. (2012). Identification of host genes linked with the survivability of chickens infected with recombinant viruses possessing $\mathrm{H} 5 \mathrm{~N} 1$ surface antigens from a highly pathogenic avian influenza virus. J. Virol. 86, 2686-2695. doi: 10.1128/JVI. 06374-11

Walden, H., and Rittinger, K. (2018). RBR ligase-mediated ubiquitin transfer: a tale with many twists and turns. Nat. Struct. Mol. Biol. 25, 440-445. doi: 10.1038/s41594-018-0063-3

Warner, J. F., and Dennert, G. (1982). Effects of a cloned cell line with NK activity on bone marrow transplants, tumour development and metastasis in vivo. Nature 300, 31-34. doi: 10.1038/300031a0

Weber, J., Polo, S., and Maspero, E. (2019). HECT E3 ligases: a tale with multiple facets. Front. Physiol. 10:370. doi: 10.3389/fphys.2019.00370

Wright, P. E., and Dyson, H. J. (2015). Intrinsically disordered proteins in cellular signalling and regulation. Nat. Rev. Mol. Cell Biol. 16, 18-29. doi: 10.1038/ nrm3920

Wu, C. H., Li, J., Li, L., Sun, J., Fabbri, M., Wayne, A. S., et al. (2019). Extracellular vesicles derived from natural killer cells use multiple cytotoxic proteins and killing mechanisms to target cancer cells. J. Extracell. Vesicles 8:1588538. doi: 10.1080/20013078.2019.1588538

Xiao, H., Shan, L., Zhu, H., and Xue, F. (2012). Detection of significant pathways in osteoporosis based on graph clustering. Mol. Med. Rep. 6, 1325-1332. doi: 10.3892/mmr.2012.1082

Yao, F., Zhou, Z., Kim, J., Hang, Q., Xiao, Z., Ton, B. N., et al. (2018). SKP2and OTUD1-regulated non-proteolytic ubiquitination of YAP promotes YAP nuclear localization and activity. Nat. Commun. 9:2269. doi: 10.1038/s41467018-04620-y

Zhou, H., Luo, Y., Mizutani, M., Mizutani, N., Reisfeld, R. A., and Xiang, R. (2005). T cell-mediated suppression of angiogenesis results in tumor protective immunity. Blood 106, 2026-2032. doi: 10.1182/blood-2005-03-0969

Conflict of Interest: The authors declare that the research was conducted in the absence of any commercial or financial relationships that could be construed as a potential conflict of interest.

Copyright (c) 2020 Lawrence, Willard, Cochran, Matchett and Kornbluth. This is an open-access article distributed under the terms of the Creative Commons Attribution License (CC BY). The use, distribution or reproduction in other forums is permitted, provided the original author(s) and the copyright owner(s) are credited and that the original publication in this journal is cited, in accordance with accepted academic practice. No use, distribution or reproduction is permitted which does not comply with these terms. 